\section{Média diária de tempo sentado \\ e fatores associados em adultos residentes no município de Ribeirão Preto-SP, 2006: Projeto OBEDIARP}

\section{Sitting-time means and correlates in adults living in Ribeirão Preto-SP, Brazil, in 2006: OBEDIARP Project}

\author{
Cláudio Shigueki Suzuki' \\ Suzana Alves de Moraes"

\section{Isabel Cristina Martins de Freitas'} \\ ' Programa de Pós-Graduação Enfermagem e Saúde Pública da Escola de \\ Enfermagem de Ribeirão Preto-Universidade de São Paulo. Núcleo de \\ Epidemiologia-NEPI-EERP-USP. \\ " Departamento de Enfermagem Materno-Infantil e Saúde Pública da Escola \\ de Enfermagem de Ribeirão Preto da Universidade de São Paulo. Núcleo de \\ Epidemiologia-NEPI-EERP-USP.
}

Fontes de Financiamento: Projeto OBEDIARP: CNPq- Edital MCT-Saúde n 30 (Processo $n^{\circ}$ 505622/2004-1); CSS - Bolsa de Doutorado CNPq, Processo n 142653/2007-1; ICMF - Bolsa de Doutorado CNPq, Processo no 141102/2007-1.

Correspondência: Suzana Alves de Moraes. Av. Bandeirantes, 3900. Campus Universitário - Ribeirão Preto - SP. CEP: 14040-902. E-mail: samoraes@usp.br

\section{Resumo}

Estimaram-se médias do tempo sentado e fatores associados, em adultos residentes em Ribeirão Preto-SP, em 2006. O estudo, de base populacional, teve delineamento transversal com amostra selecionada por conglomerados em três estágios. Em amostra de 1.205 elegíveis, 930 participaram do estudo. A variabilidade introduzida na terceira fração de amostragem foi corrigida pela atribuição de pesos amostrais que levaram em consideração a taxa de não-resposta e o número de elegíveis em cada domicílio, originando uma amostra ponderada de 2.197 participantes. Para o estudo de associação foram construídos modelos de regressão linear múltipla. Todas as estimativas foram calculadas levandose em consideração o efeito de desenho amostral. O tempo sentado médio diário foi de 280,9 minutos. Na análise multivariada permaneceram associadas ao tempo sentado as seguintes variáveis: sexo; anos de escolaridade; hábito de fumar; gasto metabólico (Mets*min*semana ${ }^{-1}$ ); no de horas de trabalho/dia e número de antecedentes de obesidade. Os resultados permitiram identificar os principais fatores associados ao tempo sentado, oferecendo subsídios para a construção de políticas públicas de promoção e prevenção em saúde.

Palavras-chave: Tempo sentado. Fatores associados. Estudos transversais. Doenças crônicas. Epidemiologia. Saúde Pública. 


\section{Abstract}

This study aimed to estimate mean daily sitting-time and to identify correlates of sitting-time in adults living in the urban area of Ribeirão Preto-SP, in 2006. A crosssectional population-based epidemiological study was carried out using three-stage cluster sampling. From a sample of 1,205 individuals, 930 attended the interviews. The variability introduced in the third sampling fraction was corrected by attributing sampling weights taking into account the non-response rate and the number of eligible units in each household, resulting in a weighted sample of 2,197 participants. To identify sitting time correlates, multiple linear regression models were used. The design effect was considered when calculating all estimates. The reported overall mean sitting-time/day was 280.9 minutes. In the multivariate analysis, the following correlates were kept in the final model: gender; years of education; smoking; me-

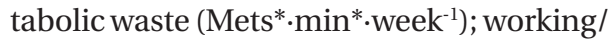
day hours, and number of familial antecedents of obesity. The results pointed out sitting-time correlates and the need for health planning related to promotion and prevention policies.

Keywords: Sitting-time. Correlates. Crosssectional studies. Chronic diseases. Epidemiology. Public Health.

\section{Introdução}

O estilo de vida sedentário é considerado uma característica marcante da sociedade contemporânea, decorrente, em grande parte, do processo de urbanização e de avanços tecnológicos, os quais se refletem diretamente em mudanças no padrão de atividade física da população, com redução do gasto energético, sobretudo em três aspectos:

- diminuição do esforço com o trabalho doméstico pelo uso de equipamentos para a execução das tarefas mais árduas;

- crescente uso da televisão como principal fonte de lazer; e

- uso do automóvel/veículo automotivo para os deslocamentos ${ }^{1,2}$.

O primeiro estudo de que se tem conhecimento, relacionando o comportamento sedentário à saúde, foi desenvolvido por Morris et al. ${ }^{3}$, em 1950, na Inglaterra, com trabalhadores. De acordo com os autores, motoristas de ônibus que permaneciam sentados durante sua jornada de trabalho apresentaram maior incidência de doença cardíaca coronariana (DCC) quando comparados aos cobradores de ônibus de dois andares, que constantemente necessitavam subir e descer as escadas do ônibus para a cobrança de bilhetes. O grau de severidade e as taxas de mortalidade por DCC foram também maiores entre aqueles que possuíam comportamento sedentário acentuado em suas ocupações.

Desde então, o estilo de vida sedentário, somado ao alcoolismo, ao tabagismo e ao padrão dietético inadequado têm sido considerados fatores de risco responsáveis pelo aumento da morbi-mortalidade por doenças crônico-degenerativas, como a obesidade, o diabetes mellitus e as doenças cardiovasculares ${ }^{2,4}$

O conceito proposto por Caspersen et al. ${ }^{5}$ sobre a "atividade física", que a definiu como qualquer movimento corporal produzido pela contração da musculatura esquelética que resulte em um gasto energético maior do que os níveis de repouso, tem sido amplamente aceito na literatura. Por 
outro lado, Varo et al. ${ }^{6}$ relataram não haver uma definição específica, aceita internacionalmente, sobre "inatividade física" ou "sedentarismo", sendo mais comum definições que incluem a participação em atividades sedentárias, como o tempo sentado, ou a "pouca/nenhuma" prática de atividade física. Para Hallal et al. ${ }^{7}$ tem sido mais comum a utilização do termo "sedentarismo" para indicar baixos níveis de atividade, sendo também empregados outros termos como "atividade física insuficiente" e "atividade física irregular". Para estes autores as várias definições propostas até o presente impedem o consenso sobre a terminologia mais adequada para indicar sedentarismo, dificultando as comparações de resultados.

Sugiyama et al. ${ }^{8}$ investigaram a relação entre o tempo sentado assistindo televisão e o tempo despendido em outros comportamentos sedentários, em uma amostra de 2.046 adultos australianos, encontrando uma associação positiva entre tais comportamentos apenas no grupo de mulheres. $\mathrm{Hu}$ et al. ${ }^{9}$, utilizando dados do Nurses'Health Study, verificaram que, independentemente do nível de atividade física, o tempo sentado esteve associado a um risco elevado de obesidade e diabetes tipo 2 .

O tempo sentado tem sido considerado como um dos principais componentes do comportamento sedentário ${ }^{2,4,4,8,9}$. Entretanto, poucos estudos de base populacional que investigaram o tempo sentado a partir de questionários padronizados e validados têm sido conduzidos em países em desenvolvimento. E, nestes casos, menos ainda se tem investigado sobre os fatores associados ao tempo dedicado às atividades sedentárias ${ }^{10}$.

Levando em consideração que a compreensão das características do comportamento sedentário é de fundamental importância para o desenvolvimento de estratégias e políticas públicas de promoção e prevenção em saúde, o presente estudo teve por objetivo avaliar a média diária de tempo sentado e identificar fatores associados em adultos residentes no município de Ribeirão Preto-SP, em 2006.

\section{Material e Métodos}

Os dados do presente estudo são provenientes do Projeto OBEDIARP, estudo epidemiológico de base populacional com delineamento transversal, intitulado "Fatores de risco para o sobrepeso, a obesidade e o diabetes mellitus no município de Ribeirão Preto, SP, 2006" ${ }^{11}$. A amostra foi composta por adultos com 30 anos e mais, de ambos os sexos, residentes em área urbana do município de Ribeirão Preto-SP, em 2006. O processo de amostragem foi desenvolvido em três estágios e a precisão das estimativas, calculadas em amostra de 1.205 indivíduos, correspondeu a erros de amostragem fixados em torno de $2 \%$ (para prevalências abaixo de $15 \%$ ou acima de $75 \%$ ) e em torno de 3\% (para prevalências entre 20 e $80 \%)$. O setor censitário foi a Unidade Primária de Amostragem (IBGE-2000), e o domicílio e as pessoas foram as unidades sorteadas, no segundo e terceiro estágios, respectivamente. O método de sorteio por conglomerados, sob partilha proporcional ao tamanho, foi adotado nos dois primeiros estágios. No terceiro estágio, sorteou-se uma pessoa com 30 anos ou mais entre os (N) residentes nos domicílios sorteados. No segundo estágio de sorteio, foi introduzida a estratificação por renda nominal média do chefe da família. No terceiro estágio, a estratificação levou em consideração a composição etária da população, segundo o sexo ${ }^{12}$, selecionando apenas 01 participante por domicílio, tendo em vista que, além das estimativas de prevalência, houve interesse em testar associações. Tal estratégia tem sido recomendada quando, diante de proposta analítica, a seleção de mais de 01 participante por domicílio pode condicionar níveis semelhantes de exposição a alguns fatores de risco/proteção, em relação aos desfechos de interesse, reforçando-se, inadequadamente, a hipótese nula ${ }^{13}$. Na presença de 02 ou mais elegíveis no domicílio, selecionou-se aquele com data de nascimento mais próxima à data da entrevista ${ }^{12}$. Ao todo foram entrevistados 930 participantes, sendo a taxa de 
resposta equivalente a 78\% [(930/(1205 - 8)] $\mathrm{x} 100$, considerando-se que, entre a data de recrutamento e a data da entrevista, 08 mulheres estavam em período de gestação ou até o sexto mês de puerpério (critério de exclusão do estudo). As perdas do estudo ( $\mathrm{n}=263$ ) foram distribuídas da seguinte forma: $6,6 \%$ por mudanças de endereço, $0,6 \%$ por óbitos e $14,6 \%$ por recusas, estas últimas, após seis tentativas de contato, em dias e horários alternados. A variabilidade introduzida, principalmente na terceira fração de amostragem, foi corrigida pela atribuição de pesos amostrais que levaram em consideração a taxa de não resposta e o número de unidades elegíveis em cada domicílio, originando uma amostra ponderada de 2.197 participantes com 30 anos e mais, de ambos os sexos, residentes na área urbana do município de Ribeirão Preto-SP, em 2006. Detalhamentos sobre o processo de amostragem, e as técnicas utilizadas para a ponderação da amostra no Projeto OBEDIARP encontram-se em Moraes et al. ${ }^{11}$. Para a aferição do tempo sentado total na semana, em minutos/dia (variável dependente) foi utilizado o International Physical Activity Questionnaire (IPAQ), versão curta, recomendado pela OMS para estudos de base populacional ${ }^{14,15}$. Quatro participantes foram excluídos do estudo, sendo dois cadeirantes e dois que apresentaram valores aberrantes para unidades de gasto metabólico (METs*minutos*semana ${ }^{-1}$ ).

As variáveis independentes foram agrupadas em 3 blocos:

- Variáveis socioeconômicas e demográficas: sexo (foram considerados ambos os sexos); idade, em anos completos, por meio do cálculo: [(data da entrevista - data de nascimento)/365,25], sendo a data de nascimento comprovada mediante a apresentação de documento de identidade ou similar. O estado marital foi classificado em 2 categorias: "vive sem companheiro" e "vive com companheiro"; nível educacional classificado segundo o número de anos completos de aprovação na escolaridade formal; e a renda do participante, que foi definida pelo valor recebido, em Reais, no mês que antecedeu a entrevista.

- Variáveis comportamentais: o hábito de fumar foi classificado em três categorias ("não fumantes", "ex-fumantes" e "fumantes") sendo a primeira a categoria de referência. Para os fumantes, considerou-se também a duração do hábito, em anos completos. Para a avaliação da dependência de bebidas alcoólicas foi aplicado o Questionário "Alcohol Use Disorders Identification Test"-AUDIT, recomendado pela OMS para estudos epidemiológicos ${ }^{16}$. A partir dos escores obtidos, utilizou-se o ponto de corte "> 8" para classificar os participantes em "não-dependentes" e "dependentes" ${ }^{17}$. Para as informações sobre o consumo de medicamentos utilizou-se como referência os últimos 15 dias que antecederam a entrevista, sendo questionados o uso e a quantidade de medicamentos utilizados, comprovados mediante a apresentação das embalagens. A variável $n^{\circ}$ de horas de trabalho/dia foi definida, segundo questão específica do Questionário $\mathrm{IPAQ}^{14}$, que inclui também o tempo dedicado ao trabalho doméstico. A atividade física foi avaliada por meio do total de unidades de gasto metabólico (METs*minutos*semana ${ }^{-1}$ ). Para o cálculo dos METs, em cada um dos três domínios, foram utilizadas as seguintes fórmulas:

Caminhada $=3,3$ METs $*$ minutos de caminhada $*$ dias de caminhada

Atividades Moderadas $=4,0$ METs * minutos de at. moderada ${ }^{*}$ dias de at. moderada

Atividades Vigorosas = 8,0 METs * minutos de at. vigorosa * dias de at. vigorosa A somatória dos valores destes três domínios resultou nos METs totais de gasto metabólico na semana. Dois casos para os quais a somatória do tempo de atividade em todos os domínios excedeu 960 minutos/semana foram excluídos da análise (valores aberrantes) ${ }^{15}$.

- Variáveis relacionadas à saúde: o estado nutricional foi classificado em 5 níveis, 
segundo pontos de corte específicos do índice de massa corporal (IMC) recomendados pela $\mathrm{OMS}^{18}$. O estado de saúde auto-referido foi definido segundo a percepção do participante sobre o seu estado de saúde e, também, comparando-a com a de seus familiares e amigos da mesma idade ${ }^{19}$. Os participantes foram também questionados sobre o número de antecedentes familiares de obesidade, incluindo irmãos, e até a terceira geração de ascendentes. Em participantes com 80 anos e mais, e desde que houvesse dúvidas decorrentes de viés de memória, os entrevistadores foram treinados para confirmarem as respostas consultando parentes ou cuidadores.

A coleta dos dados foi realizada mediante a aplicação de entrevistas estruturadas, aplicadas nos domicílios dos elegíveis por uma equipe de entrevistadores previamente treinada. Para avaliar a calibração dos entrevistadores, medidas obtidas de voluntários, durante a fase de treinamento, foram duplamente aferidas pelos entrevistadores e por um antropometrista supervisor, com experiência comprovada para estas aferições e que foi considerado o padrão ouro ${ }^{20}$. Medidas de precisão e exatidão foram calculadas, segundo a proposta de Habicht ${ }^{21}$.

Para minimizar erros de digitação, os campos de entradas de dados foram validados, através da construção de amplitudes específicas para os dígitos (intervalos de valores aceitáveis e códigos válidos para cada variável). Para garantia de qualidade no processo de digitação, os digitadores foram previamente treinados e, para o teste de consistência externa, a digitação dos dados foi efetuada através de dupla entrada de dados, em bancos formatados no software Epidata, vs. 2.1 para Windows.

A análise estatística dos dados foi desenvolvida em duas fases:

- Fase descritiva: o tempo sentado foi avaliado por meio do cálculo das médias, em minutos/dia, e intervalos com $95 \%$ de confiança. As médias, estratificadas por sexo, foram calculadas segundo as categorias das variáveis socioeconômicas, demográficas, comportamentais e relacionadas à saúde;

- Fase analítica: para a identificação dos fatores associados ao tempo sentado foram construídos modelos de regressão linear múltipla ${ }^{22}$, obtendo-se os coeficientes angulares $(\beta)$ e respectivos intervalos, com $95 \%$ de confiança, em modelos uni e multivariados.

A escolha dessa técnica estatística foi possível porque a variável tempo sentado (variável-dependente) apresentou distribuição normal ${ }^{22}$. Modelos univariados foram construídos, contendo cada uma das variáveis independentes e a variável-resposta. Nestes modelos, as variáveis para as quais se obtiveram valores $\mathrm{p} \leq 0,25$ (teste deWald) foram candidatas aos modelos multivariados ${ }^{22}$. A ordem de entrada das variáveis no modelo multivariado foi estabelecida a partir da magnitude da correlação de Pearson (matriz de correlação) entre as variáveis independentes e a variável-resposta, sendo em seguida aplicada a estratégia forward ${ }^{22}$. No modelo final permaneceram as variáveis que apresentaram valores $\mathrm{p}<0,05$. Para o modelo final foi realizada a análise de resíduos com o propósito de se verificar o pressuposto da homocedasticidade ${ }^{22}$.

Todas as estimativas foram calculadas levando-se em consideração o efeito de desenho amostral ${ }^{12}$. As análises foram efetuadas utilizando-se comandos específicos (survey commands) do software Stata, versão 8.2 para Windows, por se tratarem de comandos apropriados para o cálculo de variâncias em amostras complexas.

O Projeto OBEDIARP foi aprovado pelo Comitê de Ética em Pesquisa da Escola de Enfermagem de Ribeirão Preto-USP, em 20 de abril de 2005, e protocolado sob o $\mathrm{n}^{\circ} 0528 / 2005$. Todos os participantes assinaram o Termo de Consentimento Livre e Esclarecido, conforme recomendações da Resolução 196 do Conselho Nacional de Saúde-CNS.

Os autores declaram não existir conflitos de interesse potenciais ou reais para a realização do presente trabalho. 


\section{Resultados}

Em relação às características da amostra, foi possível verificar que $62,2 \%$ dos homens e $59,3 \%$ das mulheres estavam concentrados nas faixas etárias entre $30 \mathrm{e}$ 49 anos (Tabela 1). Quanto ao estado marital, constatou-se, em ambos os sexos, que $73,0 \%$ dos homens e $70,5 \%$ das mulheres viviam com companheiro(a). Destaca-se a baixa frequência de participantes que não frequentaram a escola ou não completaram 01 ano de escolaridade formal. Pouco mais da metade da população do estudo $(54,6 \%$ e $53,0 \%$ para homens e mulheres, respectivamente), relataram entre 08 anos completos ou mais de escolaridade. Em relação à renda do entrevistado, chama a atenção o fato de que, entre os homens, a maior frequência $(37,2 \%)$ foi encontrada entre aqueles que recebiam R $\$ 1.100,00$ ou mais. Entre as mu- lheres, $45,1 \%$ não possuíam renda e $24,4 \%$ recebiam valores inferiores a $\mathrm{R} \$ 520,00 \mathrm{de}$ renda mensal.

As Tabelas 2, 3 e 4 exibem a média diária do tempo sentado (deff $=1,1701$ ), segundo as variáveis socioeconômicas e demográficas, comportamentais e relacionadas à saúde. Em quase todas as categorias destas variáveis, os homens apresentaram valores médios de tempo sentado maiores que as mulheres, sendo que, para o conjunto dos homens, a média diária do tempo sentado foi de 306,2 minutos [IC 95\%, 283,8 - 328,6] e, para as mulheres, 270,3 minutos [IC 95\%, 256,3 - 284,2].

Em relação às variáveis socioeconômicas e demográficas (Tabela 2), no sexo masculino, a menor média diária de tempo sentado (252,6 minutos) ocorreu entre os classificados no primeiro tercil de renda $(<$ $\mathrm{R} \$ 520,00)$, enquanto que, no sexo feminino,

Tabela 1 - Características da população do estudo, segundo o sexo. Ribeirão Preto - SP, 2006. Projeto OBEDIARP.

Table 1 - Characteristics of the study population by gender. Ribeirão Preto-SP, 2006. OBEDIARP Project.

\begin{tabular}{|c|c|c|c|c|}
\hline & \multicolumn{2}{|c|}{ MASCULINO } & \multicolumn{2}{|c|}{ FEMININO } \\
\hline & $\mathbf{N}^{*}$ & $\%\left[\mathrm{IC}_{95 \%}\right]^{* *}$ & $\mathbf{N}^{*}$ & $\%\left[\mathrm{IC}_{95 \%}\right]^{* *}$ \\
\hline \multicolumn{5}{|l|}{ Faixa Etária (anos) † } \\
\hline $30-39$ & 105 & $38,0[32,3-44,1]$ & 173 & $26,3[22,2-31,0]$ \\
\hline $40-49$ & 69 & $24,2[20,0-29,1]$ & 217 & $33,0[29,6-36,5]$ \\
\hline $50-59$ & 54 & $19,0[14,9-24,0]$ & 142 & $21,4[17,9-25,4]$ \\
\hline $60+$ & 49 & $11,5[8,1-16,0]$ & 121 & $12,4[9,9-15,5]$ \\
\hline \multicolumn{5}{|l|}{ Estado Marital } \\
\hline Sem companheiro & 96 & $34,9[28,5-41,9]$ & 228 & $34,5[30,6-38,6]$ \\
\hline Com companheiro & 181 & $65,1[58,1-71,5]$ & 425 & $65,5[61,4-69,4]$ \\
\hline \multicolumn{5}{|l|}{ Nível Educacional } \\
\hline Analfabeto, $<1$ ano & 11 & $3,6[2,0-6,5]$ & 25 & $3,7[2,6-5,3]$ \\
\hline 1-3 anos & 31 & $10,5[7,5-14,5]$ & 86 & $12,5[9,9-15,8]$ \\
\hline 4-7 anos & 89 & $31,3[25,7-37,5]$ & 207 & $30,8[26,7-35,2]$ \\
\hline $8+$ & 146 & $54,6[47,5-61,3]$ & 335 & $53,0[47,9-58,1]$ \\
\hline \multicolumn{5}{|c|}{ Renda em Reais (tercis) † } \\
\hline$<520,00$ & 26 & $9,5[5,8-15,1]$ & 166 & $24,4[20,4-29,0]$ \\
\hline $520,00-1100,00$ & 97 & $33,1[26,7-40,2]$ & 97 & $14,7[12,3-17,6]$ \\
\hline$>1100,00$ & 98 & $37,2[30,6-44,3]$ & 97 & $15,8[12,2-20,3]$ \\
\hline Sem renda & 56 & $20,2[16,0-25,1]$ & 293 & $45,1[40,5-49,7]$ \\
\hline
\end{tabular}

* Número observado. Observed number

** Estimativas ponderadas considerando-se o efeito de desenho amostral. Weighed estimates considering the sample design effect.

† Valor $p<0,05$ para a estatística F. $p<0.05$ for $F$ statistics. 
Tabela 2 - Média diária do tempo sentado, segundo variáveis socioeconômicas, demográficas e sexo, com respectivos intervalos de confiança (95\%). Ribeirão Preto-SP, 2006. Projeto OBEDIARP.

Table 2 - Mean daily sitting time, according to socioeconomic status and gender, with respective confidence intervals (95\%). Ribeirão Preto-SP, 2006. OBEDIARP Project.

\begin{tabular}{|c|c|c|c|c|}
\hline \multirow{3}{*}{ VARIÁVEIS } & \multicolumn{4}{|c|}{ Valores médios do tempo sentado diário em minutos } \\
\hline & \multicolumn{2}{|c|}{ MASCULINO } & \multicolumn{2}{|c|}{ FEMININO } \\
\hline & Média* & $\mathrm{IC}_{95 \%}{ }^{*}$ & Média* & $\mathrm{IC}_{95 \%}{ }^{*}$ \\
\hline \multicolumn{5}{|l|}{ Faixa etária (anos) } \\
\hline $30-39$ & 296,9 & $259,3-334,4$ & 281,1 & $258,2-304,1$ \\
\hline $40-49$ & 300,3 & $256,2-344,4$ & 262,4 & $241,4-283,3$ \\
\hline $50-59$ & 339,7 & $291,1-388,3$ & 262,1 & $236,0-288,3$ \\
\hline $60-69$ & 268,3 & $210,2-326,4$ & 250,9 & $227,0-274,8$ \\
\hline $70+$ & 346,9 & $264,2-429,6$ & 326,9 & $272,4-381,5$ \\
\hline \multicolumn{5}{|l|}{ Estado marital } \\
\hline Sem Companheiro & 292,2 & $256,0-328,4$ & 286,8 & $262,7-310,9$ \\
\hline Com Companheiro & 309,2 & $282,7-335,8$ & 257,3 & $242,4-272,2$ \\
\hline \multicolumn{5}{|l|}{ Escolaridade (anos completos) } \\
\hline Analfabeto, $<1$ ano & 326,6 & $179,9-473,2$ & 220,9 & $155,7-286,1$ \\
\hline $1-3$ anos & 327,0 & $257,1-396,8$ & 260,2 & $229,5-290,9$ \\
\hline 4-7 anos & 265,8 & $232,3-299,3$ & 247,6 & $225,6-269,6$ \\
\hline $8+$ anos & 323,4 & $293,0-353,8$ & 289,3 & $273,7-304,9$ \\
\hline \multicolumn{5}{|l|}{ Renda individual (em R\$) } \\
\hline Sem renda & 375,7 & $313,5-438,0$ & 271,1 & $253,3-288,8$ \\
\hline $1^{\circ}$ tercil $(<520,00)$ & 252,6 & $182,9-322,3$ & 243,5 & $218,3-268,7$ \\
\hline $2^{\circ}$ tercil $(520,00-1.100,00)$ & 266,8 & $236,1-297,5$ & 252,5 & $219,8-285,2$ \\
\hline $3^{\circ}$ tercil $(>1.100,00)$ & 317,8 & $277,8-357,7$ & 325,8 & $295,2-356,4$ \\
\hline Total & 306,2 & $283,8-328,6$ & 270,3 & $256,3-284,2$ \\
\hline
\end{tabular}

* Estimativas ponderadas considerando-se o efeito de desenho amostral. Weighed estimates considering the sample design effect.

a menor média foi observada entre aquelas classificadas como analfabetas $(220,9 \mathrm{mi}-$ nutos). A maior média de tempo sentado diário no sexo masculino foi detectada entre os participantes que não referiram renda (375,7 minutos), e no sexo feminino entre aquelas com 70 anos e mais $(326,9$ minutos). A transformação destes minutos em horas de tempo sentado equivale, em média, a 6 horas e 15 minutos/dia para o sexo masculino e a 5 horas e 27 minutos/ dia para o sexo feminino. Quanto à escolaridade, destaca-se, no sexo masculino, que aqueles que completaram de 4 a 7 anos de escolaridade apresentaram um valor médio de tempo sentado diário inferior, em aproximadamente 60 minutos, em relação aos demais estratos desta variável. Entre os participantes que referiram renda mensal, observou-se, em ambos os sexos, uma relação direta entre a renda e a média diária de tempo sentado.

Em relação às variáveis comportamentais (Tabela 3), observou-se, em ambos os sexos, que os fumantes apresentaram uma média diária de tempo sentado maior que os não fumantes. Em relação ao uso de medicamentos, os homens usuários apresentaram maior média diária de tempo sentado que os não usuários. Em ambos os sexos, a menor média diária de tempo sentado foi observada entre aqueles classificados acima da mediana do gasto metabólico (METs*min*semana ${ }^{-1}$ ), podendo-se identificar, entre os homens, 
que a diferença na média diária de tempo sentado foi, aproximadamente, 72 minutos. Homens e mulheres classificados no $1^{\circ}$ tercil da variável $n^{\circ}$ de horas/dia dedicadas ao trabalho obtiveram as maiores médias diárias de tempo sentado, que corresponderam, respectivamente, a 330,3 e 288,7 minutos.

Em relação às variáveis relacionadas à saúde (Tabela 4), destaca-se, no sexo masculino, indicação de gradiente linear da média diária de tempo sentado, nas categorias do estado nutricional, com diferença entre os limites extremos de aproximadamente 200 minutos, ou 3 horas e 20 minutos. Quanto à saúde auto-referida, tanto no sexo masculino como no feminino, as maiores médias diárias de tempo sentado (425,8 e 291,7 minutos, respectivamente) foram observadas entre aqueles que consideraram sua saúde como ruim/péssima. Ao compararem seu estado de saúde com o de familiares da mesma idade, homens e mulheres classificados na categoria "igual à sua” apresentaram as menores médias de tempo sentado (300,7 e 264,3 minutos, respectivamente). Entre as mulheres, houve

Tabela 3 - Média diária do tempo sentado, segundo variáveis comportamentais e sexo, com respectivos intervalos de confiança (95\%). Ribeirão Preto-SP, 2006. Projeto OBEDIARP.

Table 3 - Mean daily sitting time according to behavioral variables and gender, with respective confidence intervals (95\%). Ribeirão Preto-SP, 2006. OBEDIARP Project.

\begin{tabular}{|c|c|c|c|c|}
\hline \multirow{3}{*}{ VARIÁVEIS } & \multicolumn{4}{|c|}{ Valores médios do tempo sentado diário em minutos } \\
\hline & \multicolumn{2}{|c|}{ MASCULINO } & \multicolumn{2}{|c|}{ FEMININO } \\
\hline & Média* & $I C_{95 \%}^{*}$ & Média* & $\mathrm{IC}_{95 \%}{ }^{*}$ \\
\hline \multicolumn{5}{|l|}{ Hábito de fumar } \\
\hline Não fumantes & 301,9 & $269,9-334,0$ & 263,5 & $246,6-280,5$ \\
\hline Ex-fumantes & 305,9 & $265,6-346,1$ & 277,3 & $247,5-307,2$ \\
\hline Fumantes & 313,4 & $270,2-356,7$ & 285,0 & $254,9-315,2$ \\
\hline \multicolumn{5}{|l|}{ Tempo de fumo } \\
\hline $1 \circ$ tercil (<13 anos) & 299,9 & $255,7-344,2$ & 278,0 & $239,3-316,7$ \\
\hline $2^{\circ}$ tercil (13-24 anos) & 284,6 & $235,6-333,7$ & 315,8 & $271,9-359,6$ \\
\hline $3^{\circ}$ tercil $(25$ anos e +$)$ & 337,4 & $282,2-392,6$ & 252,8 & $228,9-276,8$ \\
\hline \multicolumn{5}{|l|}{ Escores do AUDIT } \\
\hline Sem Dependência (0-7) & 332,4 & $303,3-361,4$ & 270,2 & $255,4-284,9$ \\
\hline Com Dependência (8-40) & 272,4 & $245,3-299,5$ & 271,1 & $241,1-301,2$ \\
\hline \multicolumn{5}{|l|}{ Uso de medicamentos } \\
\hline Não & 282,8 & $246,6-319,0$ & 276,9 & $244,4-309,3$ \\
\hline Sim & 319,1 & $291,3-346,8$ & 269,1 & $254,3-283,9$ \\
\hline \multicolumn{5}{|l|}{ Número de medicamentos } \\
\hline Nenhum & 282,8 & $246,6-319,0$ & 276,9 & $244,4-309,3$ \\
\hline 1 & 310,3 & $266,9-353,6$ & 245,7 & $223,1-268,3$ \\
\hline 2 & 343,7 & $278,4-408,9$ & 270,4 & $241,1-299,6$ \\
\hline $3+$ & 311,7 & $259,2-364,2$ & 285,7 & $265,4-306,0$ \\
\hline \multicolumn{5}{|c|}{ Gasto metabólico (METs*min.* semana-1) } \\
\hline$\leq$ mediana $(412,50)$ & 341,9 & $306,1-377,8$ & 280,2 & $261,4-299,0$ \\
\hline acima da mediana & 270,1 & $247,0-293,2$ & 260,1 & $240,4-279,8$ \\
\hline \multicolumn{5}{|l|}{ No horas/ dia de trabalho } \\
\hline $1^{\circ}$ tercil $(<7)$ & 330,3 & $285,9-374,8$ & 288,7 & $271,7-305,7$ \\
\hline $2^{\circ}$ tercil $(7-10)$ & 286,3 & $254,0-318,5$ & 252,9 & $235,1-270,7$ \\
\hline $3^{\circ}$ tercil $(>10)$ & 309,0 & $266,6-351,5$ & 274,3 & $242,9-305,7$ \\
\hline Total & 306,2 & $283,8-328,6$ & 270,3 & $256,3-284,2$ \\
\hline
\end{tabular}

* Estimativas ponderadas considerando-se o efeito de desenho amostral. Weighed estimates considering the sample design effect. 
Tabela 4 - Média diária do tempo sentado, segundo variáveis relacionadas à saúde e sexo, com respectivos intervalos de confiança (95\%). Ribeirão Preto-SP, 2006. Projeto OBEDIARP.

Table 4 - Mean daily sitting time according to health-related variables and gender, with respective confidence intervals (95\%). Ribeirão Preto-SP, 2006. OBEDIARP Project.

\begin{tabular}{|c|c|c|c|c|}
\hline \multirow{3}{*}{ VARIÁVEIS } & \multicolumn{4}{|c|}{ Valores médios do tempo sentado diário em minutos } \\
\hline & \multicolumn{2}{|c|}{ MASCULINO } & \multicolumn{2}{|c|}{ FEMININO } \\
\hline & Média* & $\mathrm{IC}_{95 \%}{ }^{*}$ & Média* & $\mathrm{IC}_{95 \%}{ }^{*}$ \\
\hline \multicolumn{5}{|l|}{ Estado nutricional } \\
\hline Normal $(18,50-24,99)$ & 286,2 & $251,8-320,6$ & 262,6 & $244,5-280,6$ \\
\hline Pré-obeso $(25,00-29,99)$ & 308,7 & $274,2-343,2$ & 273,4 & $252,6-294,1$ \\
\hline Obeso $1(30,00-34,99)$ & 316,3 & $256,5-376,1$ & 272,6 & $246,8-298,4$ \\
\hline Obeso $2(35,00-39,99)$ & 363,6 & $226,6-500,7$ & 319,8 & $240,5-399,1$ \\
\hline Obeso $3(\geq 40,00)$ & 486,5 & $307,5-665,5$ & 269,1 & $216,5-321,6$ \\
\hline \multicolumn{5}{|l|}{ Saúde auto-referida } \\
\hline Excelente & 311,2 & $273,4-349,0$ & 287,1 & $251,6-322,7$ \\
\hline Boa & 302,7 & $272,9-332,5$ & 266,3 & $247,2-285,3$ \\
\hline Regular & 291,3 & $242,8-339,8$ & 263,6 & $244,0-283,1$ \\
\hline Ruim /Péssima & 425,8 & $288,8-562,7$ & 291,7 & $247,2-336,2$ \\
\hline \multicolumn{5}{|c|}{ Saúde em relação aos familiares } \\
\hline Pior que a sua & 317,2 & $273,4-361,1$ & 269,4 & $248,6-290,1$ \\
\hline Igual a sua & 300,7 & $275,8-325,6$ & 264,3 & $245,3-283,3$ \\
\hline Melhor que a sua & 303,4 & $208,9-397,9$ & 290,8 & $259,8-321,7$ \\
\hline \multicolumn{5}{|c|}{ Saúde em relação aos amigos } \\
\hline Pior que a sua & 271,5 & $219,5-323,4$ & 279,5 & $250,6-308,4$ \\
\hline Igual a sua & 320,4 & $292,6-348,2$ & 264,0 & $243,3-284,7$ \\
\hline Melhor que a sua & 304,8 & $262,7-346,8$ & 273,7 & $253,4-294,0$ \\
\hline \multicolumn{5}{|c|}{$N^{\circ}$ de antecedentes familiares de obesidade } \\
\hline Nenhum & 304,8 & $276,1-333,4$ & 248,5 & $232,7-264,3$ \\
\hline Com Apenas 1 & 322,6 & $284,5-360,8$ & 274,9 & $251,6-298,2$ \\
\hline Com 2 ou + & 273,9 & $226,6-321,1$ & 326,0 & $296,3-355,7$ \\
\hline Total & 306,2 & $283,8-328,6$ & 270,3 & $256,3-284,2$ \\
\hline
\end{tabular}

* Estimativas ponderadas considerando-se o efeito de desenho amostral. Weighed estimates considering the sample design effect.

indicação de gradiente linear para a média de tempo sentado, nas categorias da variável $\mathrm{n}^{\mathrm{o}}$ antecedentes familiares de obesidade.

Na Tabela 5, apresentam-se os valores brutos e ajustados dos coeficientes angulares $(\beta)$ para as variáveis remanescentes no modelo final, representadas por sexo; escolaridade; hábito de fumar; gasto metabólico (METs*min*semana ${ }^{-1}$ ), $\mathrm{n}^{\mathrm{o}}$ de horas/ dia dedicadas ao trabalho e antecedentes familiares de obesidade. As variáveis sexo, gasto metabólico e n ${ }^{\circ}$ de horas/dia dedica- das ao trabalho apresentaram uma relação inversa com o desfecho. O teste de adequação ao modelo linear múltiplo indicou homocedasticidade dos resíduos.

\section{Discussão}

O Instrumento IPAQ tem sido utilizado, na maioria das vezes, para avaliar o padrão de atividade física da população, por meio do cálculo de unidades de gasto metabólico (METs) aplicado às atividades diárias, bem 
Tabela 5 - Estimativas brutas e ajustadas dos coeficientes angulares ( $\beta$ ) e respectivos intervalos de confiança (95\%). Modelo final. Ribeirão Preto-SP, 2006. Projeto OBEDIARP.

Table 5 - Crude and adjusted angular coefficient estimates ( $\beta$ ) with respective confidence intervals (95\%). Final model. Ribeirão Preto, SP, 2006. OBEDIARP Project.

\begin{tabular}{|c|c|c|c|c|}
\hline VARIÁVEIS & $\beta$ bruto* & $\mathrm{IC}_{95 \%}{ }^{*}$ & $\beta$ ajustado* & $\mathrm{IC}_{95 \%}{ }^{*}$ \\
\hline \multicolumn{5}{|l|}{ Sexo } \\
\hline Masculino & 0 & & 0 & \\
\hline Feminino & $-35,928$ & $-62,102--9,754$ & $-37,661$ & $-62,338--12,984$ \\
\hline Escolaridade (anos completos) & 5,335 & $3,240-7,429$ & 5,569 & $3,416-7,722$ \\
\hline \multicolumn{5}{|l|}{ Hábito de fumar } \\
\hline Não fumante & 0 & & 0 & \\
\hline Ex-fumante & 18,348 & $-6,719-43,417$ & 11,449 & $-10,872-33,771$ \\
\hline Fumante & 23,510 & $-3,604-50,626$ & 27,146 & $1,616-52,677$ \\
\hline Gasto metabólico (METs*min.* semana-1) & $-0,008$ & $-0,014--0,002$ & $-0,008$ & $-0,014--0,002$ \\
\hline No horas/ dia de trabalho & $-4,597$ & $-8,319--0,875$ & $-6,867$ & $-10,738--2,996$ \\
\hline$N^{\circ}$ de antecedentes familiares de obesidade & 19,446 & $8,821-30,071$ & 18,775 & $6,997-30,553$ \\
\hline
\end{tabular}

* Estimativas ponderadas considerando-se o efeito de desenho amostral. Weighed estimates considering the sample design effect.

como do tempo e da frequência semanais dedicados a atividades físicas.

Embora o IPAQ tenha sido validado e recomendado pela OMS, não apenas para a aferição da atividade física, mas também para avaliação do tempo sentado, poucos autores têm utilizado este Instrumento para a aferição deste último desfecho em estudos de base populacional, destacandose o estudo conduzido por Santos et al. ${ }^{23}$. Estes autores, em estudo realizado na Ilha de Açores/Portugal, em 2004, com 7.330 residentes com 18 anos e mais, relataram que a média diária de tempo sentado para o sexo masculino foi de 262,9 minutos e, para o feminino, 244,0 minutos. No que pesem as diferenças etárias em relação àquelas consideradas no presente estudo, o padrão apresentado de tempo sentado segundo o sexo nestas populações foi semelhante.

Resultados de estudo desenvolvido por Jans et al. ${ }^{24}$, na Holanda, entre 2000 e 2005, com 7.720 trabalhadores, revelaram que a média diária de tempo sentado (no trabalho, incluindo deslocamentos e em repouso) foi de 423 minutos/dia, valor superior aos encontrados no presente estudo, tanto no sexo masculino (306,2 minutos/dia) quanto no feminino (270,3 minutos/dia). Estas diferenças poderiam ser explicadas a partir dos seguintes fatores:

- populações de referência distintas;

- instrumentos diferentes para aferir o tempo sentado e

- jornada de trabalho. A influência da jornada de trabalho ficou evidente nos trabalhadores holandeses de tempo integral que apresentaram tempo sentado de 444 minutos / dia, maior que a média geral dos trabalhadores.

Rosenberg et al. ${ }^{25}$, em estudo transversal conduzido com 289 participantes de três países (EUA, Inglaterra e Holanda), verificaram que, dentre os participantes pertencentes ao tercil mais elevado de tempo sentado, $44 \%$ foram classificados como fisicamente ativos. Isto sugere que os indivíduos, mesmo apresentando acentuado comportamento sedentário, podem ao mesmo tempo estar engajados em programas de atividade física em níveis suficientes para promover benefícios à saúde. Dessa forma, seria incorreto caracterizar indivíduos com elevado tempo sentado/dia como fisicamente inativos.

A escolha do protocolo para avaliar o "tempo sentado" ou o "sedentarismo" na população é também uma questão im- 
portante a ser considerada. Um estudo de revisão sistemática da literatura, realizado por Hallal et al. ${ }^{7}$, no qual foram analisados apenas pesquisas com dados coletados no Brasil, revelou que a prevalência de sedentarismo diferiu amplamente entre os estudos, em função dos diferentes instrumentos utilizados, oscilando entre $26,7 \%$ e $78,2 \%$. Por outro lado, os autores também constataram a utilização de 26 diferentes definições operacionais de "sedentarismo" nesses estudos. Embora a proposta do presente estudo não seja avaliar o "sedentarismo" na população de Ribeirão Preto, e sim o "tempo sentado", a escolha do IPAQ para avaliação desta condição levou em consideração ser este um Instrumento validado internacionalmente, possibilitando futuras comparações com outros estudos populacionais, mesmo que desenvolvidos em outros países.

Sugiyama et al. ${ }^{8}$, em estudo transversal conduzido entre 2003 e 2004, com amostra de 2.046 adultos residentes na área urbana de Adelaide, Austrália, detectaram médias diárias de tempo sentado, durante o tempo de lazer, correspondentes a 247,3 e 227,5 minutos/dia para os sexos masculino e feminino, respectivamente. Embora neste estudo os autores não tenham considerado o tempo sentado durante o trabalho, o tempo sentado diário, no sexo masculino, foi superior ao feminino, resultados semelhantes aos encontrados na população adulta, residente em Ribeirão Preto. Estes resultados sugerem que a avaliação do tempo sentado em diferentes domínios (lazer ou trabalho) não foram suficientes para explicar as respectivas diferenças de tempo sentado entre os sexos.

No presente estudo, uma das possíveis explicações para os achados de menor média diária de tempo sentado no sexo feminino, em relação ao masculino, encontra respaldo no fato de as mulheres se dedicarem a múltiplas jornadas de trabalho. Os resultados da Pesquisa Nacional por Amostra de Domicílios (PNAD) ${ }^{26}$, conduzida pelo IBGE no Brasil, em 2007, revelaram que $93,3 \%$ das mulheres entre 25 e 49 anos de idade, além de exercerem atividades de trabalho formal, são também responsáveis pelo trabalho doméstico. Entre os homens, este percentual foi de apenas $54 \%$, na mesma faixa etária. Na região sudeste do Brasil, a carga horária média dedicada ao trabalho doméstico, entre as mulheres, foi de 21,3 horas/semana, além das 36,7 horas/semana dedicadas ao trabalho formal. Entre os homens, os respectivos valores foram 9,0 e 44,2 horas/semana. Depreende-se, portanto, que, entre as mulheres, o maior tempo dedicado às tarefas domésticas, em relação aos homens, acrescido do tempo dedicado às atividades em trabalho formal, deve ter contribuído para a menor média diária de tempo sentado.

Em 2006 foi implantado em todas as 26 capitais brasileiras e no Distrito Federal o Sistema de Vigilância de Fatores de Risco e Proteção para Doenças Crônicas, por Inquérito Telefônico-VIGITEL ${ }^{27}$, em amostra de 54.369 pessoas. A partir dos resultados desta pesquisa, Malta et al. ${ }^{28}$ constataram que os homens brasileiros são quase duas vezes mais sedentários $(39,8 \%)$ do que as mulheres $(20,1 \%)$, em quase todas as faixas etárias. Os autores também verificaram que a prevalência de sedentarismo aumentou com a escolaridade, em ambos os sexos. Embora Malta et al. ${ }^{28}$ tenham utilizado uma definição diferenciada de sedentarismo, tais resultados se assemelham, em seu conjunto, aos encontrados em Ribeirão Preto, exceto aqueles obtidos para os homens com escolaridade entre 4 e 7 anos, que apresentaram média diária de "tempo sentado" inferior à encontrada nas demais categorias de escolaridade. Estes achados podem ser indicativos das características do município, em relação à inserção no mercado de trabalho de homens com 4 a 7 anos de escolaridade que exercem, de modo geral, atividades no comércio, na operação de máquinas da agroindústria e na prestação de serviços gerais.

Mummery et al. ${ }^{29}$, em estudo conduzido em 2003, na Austrália, com amostra de 1.579 trabalhadores, ao mensurarem o tempo sentado, segundo categorias do estado nutricional, verificaram que a média diária 
de tempo sentado foi menor entre os classificados como normais (IMC $<25 \mathrm{~kg} / \mathrm{m}^{2}$ ), em comparação com aqueles com excesso de peso (IMC $\geq 25 \mathrm{~kg} / \mathrm{m}^{2}$ ). Estes resultados também foram semelhantes aos encontrados em Ribeirão Preto, onde foi possível verificar que as menores médias diárias de tempo sentado foram observadas entre os participantes classificados como normais, quando comparados àqueles com excesso de peso.

Dentre os estudos analíticos que buscaram investigar os preditores do sedentarismo está o estudo de Mortensen et al. ${ }^{30}$, baseado em dados da coorte denominada "University of North Carolina Alumni Heart Study" (UNCAHS). Entre 1987 e 2001, em uma amostra de 4.595 participantes, os autores constataram que a variável IMC era determinante do sedentarismo em todas as faixas etárias estudadas. Ekelund et al. ${ }^{31}$, em estudo de coorte, conduzido entre 1994 e 2003, com amostra de 393 participantes do "Medical Research Council Ely Study" (MRCES) - Cambridge, também verificaram que os indicadores IMC, peso corporal, circunferência da cintura e percentual de massa gorda eram variáveis preditoras do sedentarismo, após ajustamento para sexo, idade e tempo de seguimento. No Projeto OBEDIARP, embora a média de tempo sentado em relação às categorias do estado nutricional tenha sido indicativa de gradiente linear (sexo masculino), a variável IMC não permaneceu nos modelos finais. $\mathrm{O}$ ajustamento simultâneo para ambos os sexos, bem como o efeito de confundimento exercido por outras variáveis presentes no modelo final, retiraram a significância estatística da associação em pauta. Estes diferentes resultados podem ser ainda decorrentes dos distintos constructos utilizados, pois, enquanto nos estudos de Mortensen et al. ${ }^{30} \mathrm{e}$ Ekelund et al. ${ }^{31}$ foram investigados os fatores associados ao "sedentarismo", definido como "pouca participação em atividades físicas regulares", o presente estudo investigou o "tempo sentado", considerado um dos principais componentes do comportamento sedentário ${ }^{4,6,7,8,23,24,26}$.
No estudo conduzido por Varo et al. ${ }^{6}$ na União Européia ( $n=15.239)$, os autores investigaram o efeito independente das variáveis sexo, idade, estado nutricional, nível educacional, mudança de peso nos últimos 6 meses, estado marital e hábito de fumar sobre o comportamento sedentário (variável dependente). Permaneceram no modelo final as seguintes variáveis: nível educacional, hábito de fumar, estado marital e estado nutricional, resultados semelhantes ao elenco de variáveis que no Projeto OBEDIARP permaneceram no modelo final. Yancey et al..$^{32}$, em estudo transversal realizado em Los Angeles entre 1999 e $2000(n=8.353)$, identificaram as variáveis sexo, idade, nível educacional, local de nascimento, saúde auto-referida, tempo sentado ao computador/televisão, diagnóstico de diabetes e hábito de fumar como associadas ao comportamento sedentário. Os resultados relatados porVaro et al. ${ }^{6}$ eYancey et al..$^{32}$ corroboram, pelo menos em parte, os encontrados em Ribeirão Preto, com exceção do sentido inverso da associação entre escolaridade e tempo sentado encontrado nos dois estudos supra citados.

Hallal et al. ${ }^{33}$, em 2002, em estudo transversal $(\mathrm{n}=3.182)$ avaliando a inatividade física na cidade de Pelotas-RS, revelaram que as variáveis idade, condição socioeconômica e saúde auto-referida estiveram associadas a este desfecho. Por outro lado, as variáveis sexo, cor da pele, IMC e estado marital não apresentaram associação com inatividade física. Destaca-se, entretanto, que os autores deste estudo definiram inatividade física como praticar, em média, menos que 150 minutos semanais de atividades moderadas ou vigorosas.

Torna-se oportuno salientar que diferentes expressões, definições específicas e classificações utilizadas para identificar "inatividade física", "sedentarismo" e "comportamento sedentário”, além da composição de distintos elencos de variáveis candidatas aos modelos finais, podem ter possibilitado discrepâncias em relação aos resultados encontrados em Ribeirão Preto.

O presente estudo apresenta como uma 
de suas limitações a impossibilidade de inferir causalidade e, por conseguinte, a direcionalidade das associações encontradas, em decorrência do seu delineamento transversal. Por outro lado, o IPAQ, embora validado em diferentes países, tem sido mais utilizado para a avaliação do padrão de atividade física do que do tempo sentado, dificultando comparações entre diferentes achados.

O rigor metodológico aplicado ao Projeto OBEDIARP em todas as etapas, além da taxa de resposta satisfatória $(78 \%)^{34}$, reforça a validade interna do estudo e a suposição de que seus resultados não foram decorrentes de vícios sistemáticos.

Os resultados do presente estudo permitiram identificar os principais fatores associados ao tempo sentado em adultos residentes no município de Ribeirão Preto-SP, embora a relação entre $n^{\circ}$ de antecedentes de obesidade e este desfecho, bem como o sentido da associação entre escolaridade e tempo sentado, exijam novas investigações para a identificação de sua consistência.

No Projeto OBEDIARP, dentre as variáveis que compuseram o modelo final, identificaram-se algumas passíveis de intervenção, como o hábito de fumar e o gasto metabólico decorrente da atividade física semanal (METs*min*semana ${ }^{-1}$ ). O consumo abusivo de álcool e o uso de medicamentos, embora associados ao padrão de atividade física em outros estudos ${ }^{35,36}$, não apresentaram associação com o tempo sentado.

No que pesem as limitações do presente trabalho, os resultados permitiram identificar os principais fatores associados ao tempo sentado no município de Ribeirão Preto. Estes achados podem orientar a construção de políticas públicas com vistas à diminuição do tempo sentado, contribuindo, por conseguinte, para a prevenção de doenças crônico-degenerativas.

Contribuição dos Autores: Claudio Shigueki Suzuki participou da elaboração da proposta do estudo, colaborou no levantamento bibliográfico, análise de dados, discussão dos resultados e revisão de todas as versões do manuscrito. Suzana Alves de Moraes elaborou a proposta do estudo, colaborou na análise de dados, interpretação e discussão dos resultados e revisão de todas as versões do manuscrito. Isabel Cristina Martins de Freitas participou da elaboração da proposta do estudo, colaborou no gerenciamento dos dados, análise dos dados, discussão dos resultados e revisão de todas as versões do manuscrito.

\section{Referências}

1. Mendonça CP, Anjos LA. Aspectos das práticas alimentares e da atividade física como determinantes do crescimento do sobrepeso/obesidade no Brasil. Cad Saúde Pública 2004; 20: 1-15.

2. Hamilton MT, Hamilton DG, Zderic TW. Role of low energy expenditure and sitting in obesity, metabolic syndrome, type 2 diabetes, and cardiovascular disease. Diabetes 2007; 56: 2655-67.

3. Morris JP, Heady JA, Raffle PAB, Roberts CG, Parks JW. Coronary heart-disease and physical activity of work. The Lancet 1953; 28: 1111-20.

4. Katzmarzyk PT, Church TS, Craig CL, Bouchard C. Sitting time and mortality from all causes, cardiovascular disease, and cancer. Med Sci Sports Exerc 2009; 41: 998-1005.

5. Caspersen CJ, Powell KE, Christenson GM. Physical activity, exercise, and physical fitness: definitions and distinctions for health-related research. Public Health Reports 1985; 100: 126-31.
6. Varo JJ, Martínez-Gonzalez MA, Irala-Estévez J, Kearney J, Gibney M, Martínez A. Distribution and determinants of sedentary lifestyles in the European Group. Int J Epi 2003; 32: 138-46.

7. Hallal PC, Dumith SC, Bastos JP, Reichert FF, Siqueira FV, Azevedo MR. Evolução da pesquisa epidemiológica no Brasil: revisão sistemática. Rev Saúde Pública 2007; 41: 453-60.

8. Sugiyama T, Healy GN, Dunstan DW, Salmon J, Owen N. Is television viewing time a marker of a broader pattern of sedentary behavior? Am Behav Med 2008; 35: 245-50.

9. Hu FB, Li TY, Colditz GA et al. Television watching and other sedentary behavior in relation to risk of obesity and type 2 diabetes mellitus in women. JAMA 2003; 289:1785-91

10. Booth M. Assessment of physical activity: an international perspective. Res Quart Exerc Sport 2000; 71: $114-20$ 
11. Moraes SA, Freitas ICM, Gimeno SGA, Mondini L. Prevalência de diabetes mellitus e identificação de fatores associados em adultos residentes em área urbana de Ribeirão Preto, SP, Brasil, 2006: Projeto OBEDIARP. Cad Saúde Pública 2010; 26: 929-41.

12. Silva NN. Amostragem Probabilística: um curso introdutório. São Paulo: EDUSP; 2001.

13. Szklo M, Nieto FJ. Epidemiology: Beyond the Basics. Gaithersburg: Aspen Publishers Inc; 2000.

14. Craig C, Marshall A, Sjostrom M, Bauman AE, Booth ML, Pratt M, et al. International Physical Questionnaire: 12-country reliability and validity. Med Sci Sports Exercise 2003; 35: 1381-95.

15. IPAQ, 2005. International Physical Activity Questionnaire. Guidelines for data processing and analysis of the International Physical Activity Questionnaire (IPAQ). Disponível em http://www.ipaq. ki.se/dloads/IPAQ\%20LS\%20Scoring\%20Protocols_ Nov05.pdfS. [Acessado em outubro de 2008]

16. Organización Mundial de la Salud. Cuestionario de identificación de los trastornos debidos al consumo de alcohol-AUDIT; 2001.

17. Mendonza-Sassi RA, Béria JU. Prevalence of alcohol use disorder and associated factors: a population-based study using AUDIT in southern Brazil. Addiction 2003; 98: 799-804.

18. WHO. Obesity: preventing and managing the global epidemic. Report of a WHO Consultation. WHO Technical Report Series 894. Geneva: World Health Organization; 2000.

19. Rosa TEC, Benício MHA, Latorre MRDO, Ramos LR. Fatores determinantes da capacidade funcional entre idosos. Rev Saúde Pública 2003; 37: 40-8.

20. Castro V, Moraes SA, Freitas ICM, Mondini L. Variabilidade na aferição de medidas antropométricas: comparação de dois métodos estatísticos para avaliar a calibração de entrevistadores. Rev Bras Epidemiol 2008; 11: 278-86.

21. Habicht JP. Estandarización de métodos epidemiológicos cuantitativos sobre el terreno. Bol Oficina Sanit Panam 1974; 76(5): 375-84.

22. Kleinbaum DG, Kupper LL, Muller KE, Nizam A. Applied Regression Analysis and other Multivariable Methods. Washington: Duxbury Press; 1998.

23. Santos R, Vale S, Miranda L, Mota J. Socio-demographic and perceived environmental correlates of walking in Portuguese adults - a multilevel analysis. Health \& Place 2009; 15: 1094-9.

24. Jans MP, Proper KI, Hildebrandt VH. Sedentary behavior in Dutch workers: differences between occupations and business sectors. Am J Prev Med 2007; 33: 450-54.
25. Rosenberg DE, Bull FC, Marshall AL, Sallis JF, Bauman AE. Assessment of sedentary behavior with the International Physical Activity Questionnaire. J Physical Activity Health 2008; 5: S30-44.

26. Soares C, Sabóia AL. Tempo, trabalho e afazeres domésticos: um estudo com base nos dados da Pesquisa Nacional por Amostra de Domicílios 2001 e 2005. Rio de Janeiro: IBGE, Coordenação de População e Indicadores Sociais; 2007.

27. Moura EC, Morais Neto OI, Malta DC, Moura L, Silva NN, Bernal R et al. Vigilância de fatores de risco para doenças crônicas por inquérito telefônico nas capitais dos 26 estados brasileiros e no Distrito Federal (2006). Rev Bras Epidemiol 2008; 11: S20-37.

28. Malta DC, Moura EC, Castro AM, Cruz DKA, Morais Neto OL, Monteiro CA. Padrão de atividade física em adultos brasileiros: resultados de um inquérito por entrevistas telefônicas, 2006. Epidemiol Serv Saúde 2009; 18: 7-16.

29. Mummery WK, Schofield GM, Stelle R, Eakin EG, Brown WJ. Occupational sitting time and overweight and obesity in Australian workers. Am J Prev Med 2005; 29: 91-7.

30. Mortensen LH, Siegler IC, Barefoot JC, Gronbaek $\mathrm{M}$, Sorensen TIA. Prospective association between sedentary behavior and BMI in midlife. Obesity 2006; 14: 1462-71.

31. Ekelund U, Brage S, Besson H, Sharp S, Wareham N. Time spent being sedentary and weight gain in healthy adults: reverse or bidirectional causality? Am J Clin Nutr 2008; 88: 612-7.

32. Yancey AK, Wold CM, McCarthy WJ, Weber MD, Lee B, Simon PA et al. Physical inactivity and overweight among Los Angeles county adults. Am J Prev Med 2004; 27: $146-52$.

33. Hallal PC, Victoria CG, Wells JCK, Lima RC. Physical Inactivity: prevalence and associated variables in Brazilian adults. Med Sci Sports Exerc 2003; 35: 1894-900.

34. Bland M. An Introduction to Medical Statistics. Norfolk:Oxford University Press; 2006.

35. Jylha M. Ten-year change in the use of medical drugs among the elderly-a longitudinal study and cohort comparison. J Clin Epidemiol 1994; 47: 69-79.

36. Bardel A, Wallander MA, Svardsudd K. Reported current use of prescription drugs and some of its determinants among 35 to 65-year-old women in mid-Sweden: a population-based study. J Clin Epidemiol 2000; 53: 63743.

Recebido em: 06/12/09 Versão final reapresentada em: 10/07/10 Aprovado em: 26/08/10 